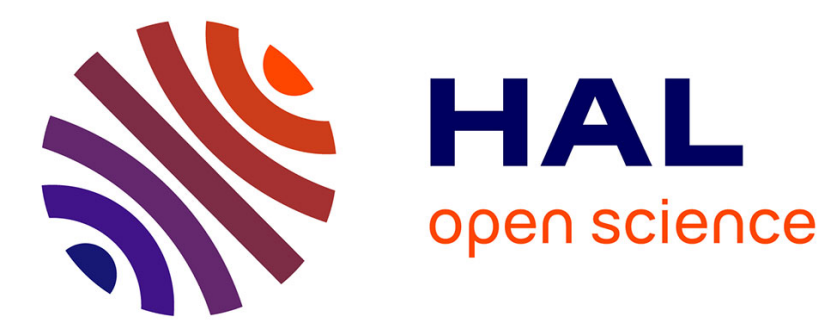

\title{
Robust Fano resonance in a topological mechanical beam
} Wei Wang, Yabin Jin, Wan Wang, Bernard Bonello, Bahram Djafari-Rouhani, Romain Fleury

\section{To cite this version:}

Wei Wang, Yabin Jin, Wan Wang, Bernard Bonello, Bahram Djafari-Rouhani, et al.. Robust Fano resonance in a topological mechanical beam. Physical Review B, 2020, 101 (2), pp.024101. 10.1103/PhysRevB.101.024101 . hal-03140628

\section{HAL Id: hal-03140628 \\ https://hal.science/hal-03140628}

Submitted on 26 Aug 2021

HAL is a multi-disciplinary open access archive for the deposit and dissemination of scientific research documents, whether they are published or not. The documents may come from teaching and research institutions in France or abroad, or from public or private research centers.
L'archive ouverte pluridisciplinaire HAL, est destinée au dépôt et à la diffusion de documents scientifiques de niveau recherche, publiés ou non, émanant des établissements d'enseignement et de recherche français ou étrangers, des laboratoires publics ou privés. 


\title{
Robust Fano resonance in a topological mechanical beam
}

\author{
Wei Wang, ${ }^{2}$ Yabin Jin $\odot,{ }^{1, *}$ Wan Wang, ${ }^{1}$ Bernard Bonello, ${ }^{2}$ Bahram Djafari-Rouhani $\odot,{ }^{3}$ and Romain Fleury ${ }^{4}$ \\ ${ }^{1}$ School of Aerospace Engineering and Applied Mechanics, Tongji University, 200092 Shanghai, China \\ ${ }^{2}$ Sorbonne Université, UPMC Université Paris 06 (INSP-UMR CNRS 7588), 4 place Jussieu 75005 Paris, France \\ ${ }^{3}$ Institut d'Electronique, de Microélectonique et de Nanotechnologie, UMR CNRS 8520, \\ Département de Physique, Université de Lille, 59650 Villeneuve d'Ascq, France \\ ${ }^{4}$ Laboratory of Wave Engineering, School of Engineering, EPFL, Station 11, 1015 Lausanne, Switzerland
}

(Received 19 November 2019; revised manuscript received 5 December 2019; published 3 January 2020)

\begin{abstract}
The advances in topological condensed matter physics enable the manipulation of classic waves in different ways, such as unidirectional propagation featuring the suppression of backscattering and the robustness against impurities and disorder, making it possible to endow classical phenomena with topological properties. Fano resonance, a widely spread and basic kind of resonance, features an asymmetric line shape with an ultrahigh quality factor $Q$ that usually requires delicate designs and precise fabrication. In this work, we achieve a robust Fano mechanical resonance with topological protection by engineering band inversion of two different vibrating symmetries of a pillared beam that gives rise to dark and bright edge modes. The Fano resonance results from the constructive and destructive interferences between topological dark and bright modes. It is further demonstrated that the Fano asymmetric shape of the transmission peak and its frequency are robust against random perturbations in the pillars' position as long as the symmetry is conserved. If random perturbations break the symmetry and only band inversion is involved, the asymmetric line shape of the Fano resonance weakens until disappearing before the closure of the bulk band gap, since the excitation will couple all fundamental modes of the beam. The analysis of the robustness of Fano resonance originating from band inversion and symmetry protection reveals the nature of topological protection which can be applied to design topological high- $Q$ resonance in sensing application.
\end{abstract}

DOI: 10.1103/PhysRevB.101.024101

\section{INTRODUCTION}

Fano resonance [1] refers to the asymmetric spectral peaks in wave scattering that widely exist in quantum systems, optics, plasmonics, photonic or phononic crystals, and metamaterials [2-6]. It can be theoretically interpreted as the coupling of two resonant states with largely different quality factors. The constructive and destructive interferences of these two resonances, each featuring a sharp spectrum, can generate an asymmetric line shape beneficial for various applications, such as beam filters, sensors, low threshold lasers, and signal communications [7-13]. However, the realization of a very high quality factor $Q$ Fano resonance poses great challenges to the fabrication processes and operating environments. In these situations, geometrical imperfections (defects, impurities, disorders, etc.) can easily occur, resulting in the shift of the resonances and the disappearance of the asymmetric line shape.

Recently, the rapid development of topological insulators [14-16], featuring both the suppression of the backscattered waves and robustness against impurities, has been extended from condensed matter physics to classical areas of physics, including acoustics and elasticity of continuous media [17-29]. Such an extension provides an alternative approach to realize robust wave guiding and topologically

*083623jinyabin@tongji.edu.cn selective wave splitting in finite-hole-drilled phononic crystal plates at Bragg frequency range [30] or in pillared phononic crystal plates in the subwavelength scale [29], which can be applied to information transmission. Topologically protected propagation can be obtained at a domain wall between insulating phononic crystals with different bulk topological properties, consistently with the well-known bulk-edge correspondence. By specifically engineering the corresponding constituents, topological edge states featuring either a high $Q$ (dark mode with a narrow-band transmission) or a low $Q$ (bright mode with a wideband transmission) can be achieved. Furthermore, by optimizing the designs in such a way that these two modes overlap in a common frequency interval, a topological Fano resonance may be formed which is immune to geometrical perturbations. The achievement in an airborne acoustic configuration was recently reported in Ref. [31]. However, the realization of topological Fano resonance in elastic media has not been explored yet, despite the fact that the topologically protected Fano resonance in elastic continuous systems is strongly desired for solid functional devices intended for signal processing and sensing [32] at the nano- and microscales. Unlike airborne sound waves with only one polarization, elastic waves in continuous media have different polarizations (e.g., four polarizations in a beam as studied in this work) which are usually coupled, making it more challenging in mode characterizations and experimental measurements. 
(a)

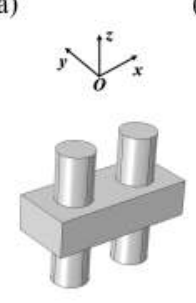

(b)

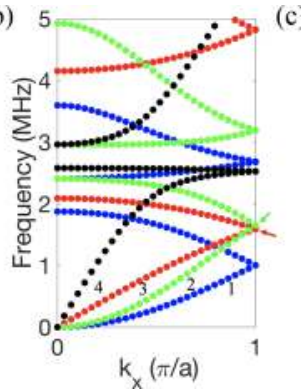

(c)

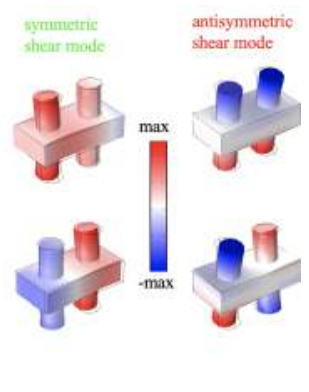

FIG. 1. (a) Unit cell of a phononic beam with the pillar spacing as $d=100 \mu \mathrm{m}$. Periodic boundary conditions along the $x$ axis are applied to the two beams' boundaries. (b) Dispersion curves of the pillared phononic beam where each color refers to a particular symmetry of the corresponding branch: band 1 (AS in blue), band 2 (SA in green), band 3 (AA in red), and band 4 (SS in black). Accidental double Dirac cones of symmetric and antisymmetric shear modes appear at $1.66 \mathrm{MHz}$ for $k_{x}=\pi / a$. (c) Real part of the displacement field $u_{y}$ for the four eigenstates at the double Dirac points as indicated by the red and green arrows.

In this work, we realize a Fano resonance with topological protection in a pillared phononic beam in an elastic continuous system. Topological band gaps for the symmetric and antisymmetric shear modes are designed by engineering a band inversion. Then a high- $Q$ (dark) and a low- $Q$ (bright) edge mode are constructed by leveraging the antisymmetry and symmetry of the beam's shear vibration, respectively. A topological Fano resonance with ultrahigh $Q$ is formed after constructive and destructive interference between the topological dark and bright modes. To probe the topological robustness, different random perturbations are introduced and the robustness of the Fano asymmetric-shape transmission line is numerically demonstrated and discussed in terms of symmetry.

\section{PILLARED PHONONIC BEAM WITH TOPOLOGICAL TRANSITION}

The considered elastic model is shown in Fig. 1(a). This is a double-sided pillared beam unit with periodic boundary conditions applied to the two lateral boundaries along the $x$ axis whose lattice constant is set to be $a=400 \mu \mathrm{m}$. The beam has a width of $200 \mu \mathrm{m}$ along the $y$ direction with a free boundary condition and a thickness of $100 \mu \mathrm{m}$ along the $z$ direction. The four identical pillars, symmetrically placed over the matrix, have a diameter of $100 \mu \mathrm{m}$ and a height of $135 \mu \mathrm{m}$. The internal distance between two pillars on each side is $d=100 \mu \mathrm{m}$. Figure 1(b) shows the corresponding band structure. The structure displays two symmetry planes, namely, $x O y$ and $x O z$, which suggests that the branches in the dispersion curves can be represented according to their symmetric (S) or antisymmetric (A) characters with respect to each of these two symmetry planes. In this respect, the four fundamental bands marked as $1-4$ can be respectively labeled as AS, SA, AA, and SS (as summarized in Table I). They correspond to the flexural, symmetric shear, antisymmetric shear (torsional), and longitudinal motions of the beam. These modes can be studied independently of each other as long

TABLE I. Symmetry properties of the four bands marked in the dispersion curves with respect to the symmetry planes $x O y$ and $x O z$. "A" and "S" are for antisymmetric and symmetric, respectively.

\begin{tabular}{lcc}
\hline \hline & $x O y$ plane & $x O z$ plane \\
\hline Band 1 (blue) & $\mathrm{A}$ & $\mathrm{S}$ \\
Band 2 (green) & $\mathrm{S}$ & $\mathrm{A}$ \\
Band 3 (red) & $\mathrm{A}$ & $\mathrm{A}$ \\
Band 4 (black) & $\mathrm{S}$ & $\mathrm{S}$ \\
\hline \hline
\end{tabular}

as the perfect symmetry of the structure is conserved, for instance, without introducing any disorder. They can also be excited independently or simultaneously depending on the symmetry of the incident wave. The introduction of disorder, as we see later, will mix some of the modes.

Two Dirac cones of the AA band 3 and SA band 2 coincide at $1.66 \mathrm{MHz}$ for $k_{x}=\pi / a$. It should be noted that exact accidental degeneracy is not crucial, but only a common band gap for bands 2 and 3 from lifting the degenerate points is required. The eigenstates (real part of displacement $u_{y}$ ) of each mode at the Dirac points are presented in Fig. 1(c), clearly illustrating the AA and SA nature of the shear modes.

Now, we set the pillar spacing in one unit cell, respectively, to $d=60 \mu \mathrm{m}$ and $d=140 \mu \mathrm{m}$ while keeping the period of the unit cell as $a=400 \mu \mathrm{m}$. The corresponding dispersion curves are displayed in Fig. 2. One can observe that the band structures in both cases are exactly the same, especially the double Dirac cones that are degenerated to form a common band gap for bands 2 and 3. The degenerated states above or below the band gap are also presented. The eigenstates above the band gap for $d=60 \mu \mathrm{m}$, shown in the red dotted box, are flipped to the eigenstates below the band gap for $d=140 \mu \mathrm{m}$. The same goes for the degenerated states in the blue dotted box. This band inversion achieved by varying the pillar spacing is symptomatic of a topological transition [33], where the Zak phase of the associated bands is identically zero for the crystal with small interval between the two pillars in the unit cell but is nonzero for the large-interval case [31]. Therefore, our system provides two different bulk structures with topological or trivial properties characterized by different symmetries in shear vibrations, which can be further used to design a Fano asymmetric line shape in the transmission spectrum.

\section{Topological interface states and topological Fano resonance}

A Fano resonance can be composed of the interaction of a dark mode and a bright mode with distinct lifetimes. If both dark and bright modes are topological edge modes, a topological Fano resonance is consequently formed. We construct a finite stripe with an interface between a topological and a trivial beam, each composed of ten units, as illustrated in Fig. 3(b). Periodic boundary conditions are applied to both edges along the $x$ axis as the period of the supercell stripe, $20 a$, is sufficiently large to calculate the interface edge states. Since the topological property is characterized by symmetry, we separately calculate the dispersion of the edge modes for symmetric (green) and antisymmetric (red) shear vibrations. We show the results in the left- and right-hand panels in 

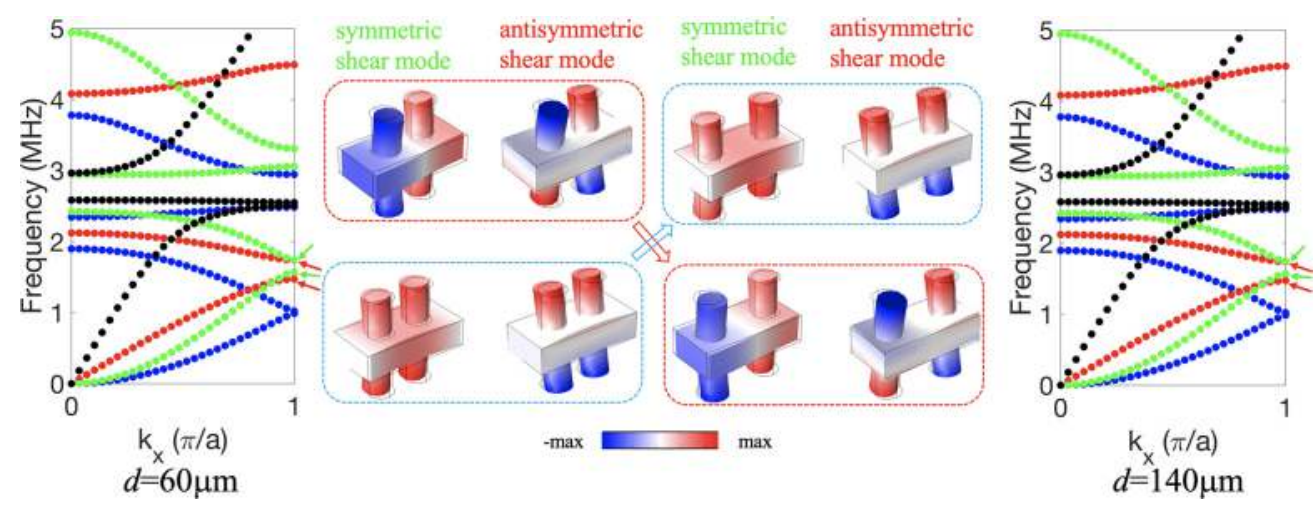

FIG. 2. Dispersion curves for the crystal with short interval $d=60 \mu \mathrm{m}$ (left-hand panel) between the pillars in the unit cell and large interval $d=140 \mu \mathrm{m}$ (right-hand panel). They show exactly the same band structure. The eigenstates of the symmetric and antisymmetric shear modes above and below the gap (shown as the green and red arrows) are presented beside the dispersion. From $d=60 \mu \mathrm{m}$ to $d=140 \mu \mathrm{m}$, the eigenstates shown in red and blue dotted boxes flip as band inversion.

Fig. 3(a), respectively. In the dispersion curves, the part in between the two shaded areas indicates the topological band gap for shear modes, containing a bright edge band and a dark edge band for symmetric and antisymmetric cases (marked as green and red), respectively. The bright edge mode shows a more pronounced dispersive character than the dark edge

(a)

(b)

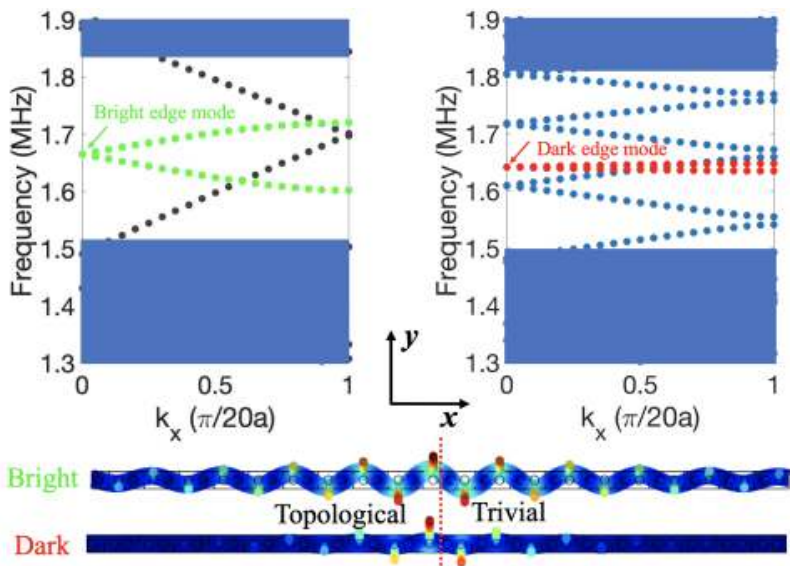

(c)

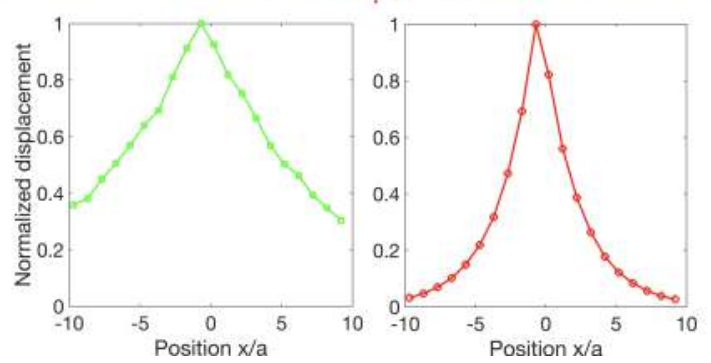

FIG. 3. A finite-length stripe consists of topological (10a) and trivial $10 a$ beams with an interface in the middle (red dotted line). The total length of the stripe is $20 a=8000 \mu \mathrm{m}$, which is sufficiently large to reveal the interface modes when periodic boundary conditions are applied to the two beam's edges along the $x$ axis. (a) The dispersion curves show bright and dark shear edge modes (in green and red) in the topological band gap, respectively. (b) The eigenstates of the two edge modes (absolute displacement $u_{y}$ ). (c) Normalized displacements in pillars along the beam for bright (left) and dark (right) edge modes. mode, resulting from a weaker confinement of the eigenstate. This clearly appears in the displacement field shown in Fig. 3(b). It is noted that the folded bulk bands in the leftand right-hand panels of Fig. 3(a) are the SS (longitudinal) and AS (flexural) modes in Fig. 1, respectively. Since their polarizations and excitations are different from shear bands 2 and 3 , the excitation of shear edge modes will not excite the modes in bands 1 and 4 in this ordered system. The designed band gaps apply only for shear modes as shown in Fig. 3(a). The displacement of the edge mode decays exponentially away from the interface with a decay factor which is inverse to the Dirac velocity [34]. From Fig. 1(b), the Dirac velocity of band 3 is smaller than that of band 2, so the decay factor for the dark edge mode is higher than that for the bright one. In Fig. 3(c), we show the normalized displacement profile of vibrating pillars along the beam, supporting the exponential attenuation away from the interface and the higher decay factor for the dark edge mode.

The bright and dark edge modes directly relate to the symmetry of the beam's shear vibrations that can be excited with different symmetries of shear forces. Line forces along the $y$ axis are applied to the top and bottom edges of the beam cross section. The bright edge state (related to the symmetric shear vibration) can be excited when the upper and lower line forces are oriented in the same direction, whereas the dark edge state (related to the antisymmetric shear vibration) can be excited when these forces are pointing in opposite directions. We consider a finite pillared beam built by merging a topological and a trivial subspace (one supercell in Fig. 3) with a length of $20 a$ in total. The transmission spectra of the two edge states are calculated and displayed in the left-hand panel of Fig. 4, where the dark-edge-mode-induced transmission peak is much sharper than its bright mode counterpart.

If a single line force is applied to either the top or the bottom edge, both symmetric and antisymmetric shear vibrations can be excited, allowing for the dark and bright edge modes to couple and to interact with each other. Such an interaction will lead to constructive and destructive interferences that further generate a Fano asymmetric line-shape transmission, as proved in the right-hand panel of Fig. 4. The Fano resonance has an extrasharp shape with an extremely high quality factor $\mathrm{Q}=f_{c} / \Delta f$ over 5000 , where $f_{c}$ and $\Delta f$ 


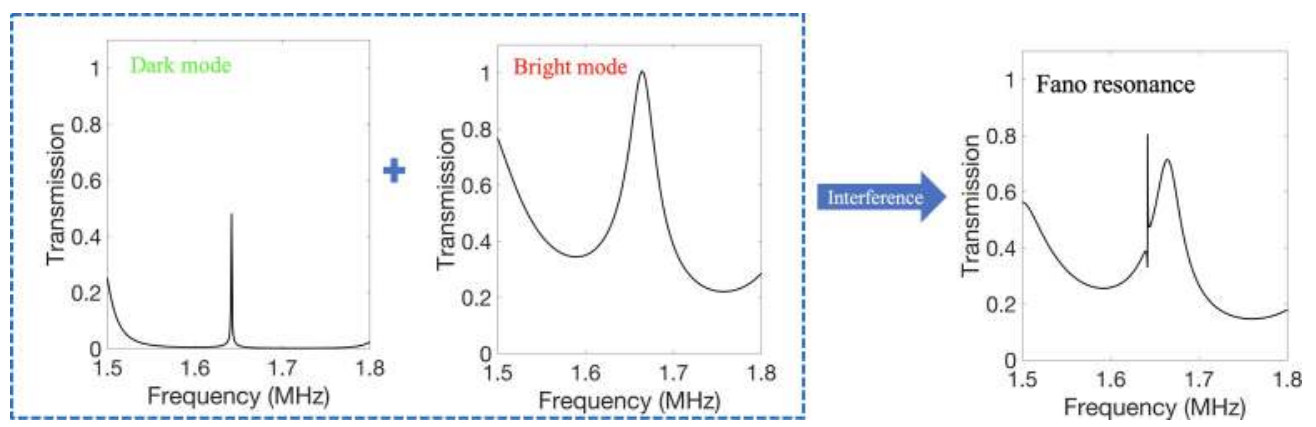

FIG. 4. Left: Dark and bright transmission spectra with single symmetric and antisymmetric shear vibration excitation, respectively. Right: When both shear vibrations are excited, the dark and bright modes will couple and interfere to generate the high- $Q$ Fano asymmetric shape transmission.

are the midfrequency and the common width of the peak and dip of the Fano resonance, respectively. Due to the high $Q$ property, the Fano resonance gets easily disturbed and loses the asymmetric line shape as a result of random perturbation or fabrication defects. However, here the dark and bright edge states are topologically protected and therefore the induced Fano resonance, being of topological nature, is robust against perturbations. It should be noted that topological Fano resonance can also be achieved by band inversion from bands 1 and 4 with optimizing parameters.

To verify the robustness of the topological Fano resonance, we introduce randomness in the pillars' position in two ways: maximum position perturbation $\delta_{x}$ along the $x$ axis and $\delta_{y}$ along the $y$ axis. The result is presented in Fig. 5. For the sake of simplicity, we keep the symmetry with respect to the middle $x O y$ plane of the beam and assume that any pair of upper and lower pillars with central symmetry moves simultaneously. For random perturbation along the $x$ axis, one can notice that the symmetry with respect to the vertical $x O z$ plane is still preserved, which means that the classification of the modes as AS, SA, AA, and SS remains unaltered. In other words, it is the symmetry of the incident wave which defines if each type of mode is excited independently of the others or simultaneously with some of them. In the upper panels of Fig. 5, one can notice that going to higher $\delta_{x}$, it decreases the peak of the dark mode which further slightly reduces the peak of Fano resonance. It is observed that the dark, bright, and Fano asymmetric shapes are always conserved and their frequencies are almost unchanged for any of the considered random perturbations $\delta_{x}$, which is of primary interest for signal information communication application in microscale. For random perturbation along the $y$ axis, the symmetry with 0000000000000000000000000000000000000000
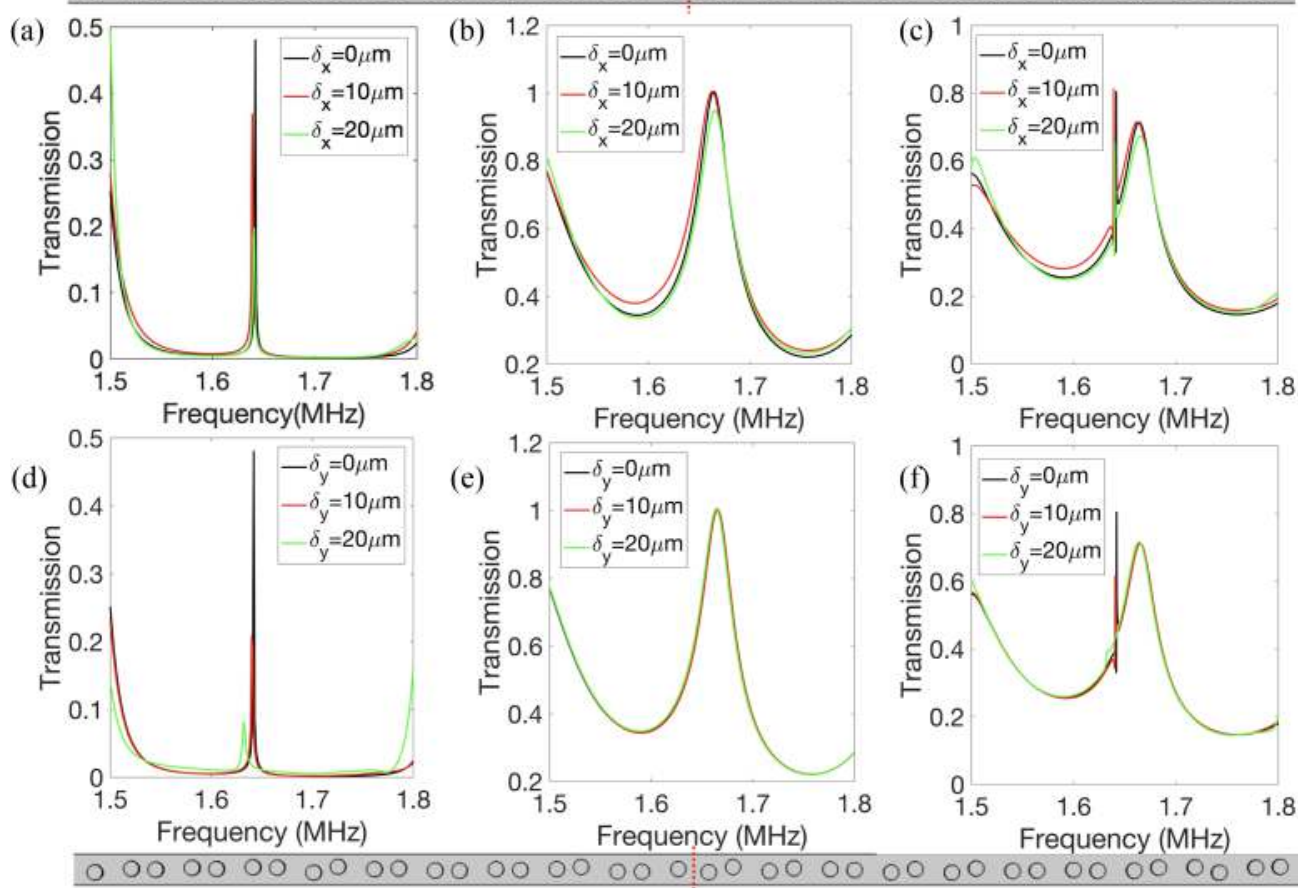

FIG. 5. Robustness of topological (a), (d) dark mode, (b), (e) bright mode, and (c), (f) Fano resonance against perturbation in the pillars' position with random degree $\delta$ along the $x$ (a)-(c) and $y$ (d)-(f) axes. The position profiles of $\delta_{x}=20 \mu \mathrm{m}$ and $\delta_{y}=20 \mu \mathrm{m}$ are shown at the top and bottom, respectively. 
respect to the $x O z$ plane is broken, so one can expect a coupling between modes 1 and 3 (originally AS and AA) and between modes 2 and 4 (originally SA and SS). Therefore, in Fig. 5(d) the excitation of the dark torsional mode 3 by means of an antisymmetric shear wave produces a coupling with the flexural mode 1 and one observes that increasing $\delta_{y}$ up to $20 \mu \mathrm{m}$ significantly decreases the peak of the dark mode and also slightly downshifts its frequency. Meanwhile, the excitation of the bright mode in Fig. 5(e) by means of a symmetrical shear wave produces a coupling with the longitudinal mode 4 but almost no influence is observed on the bright mode, presumably due to the different nature of modes 2 and 4 and also to the already broad bright mode. Finally, in Fig. 5(f), both dark and bright modes are excited simultaneously, which results in a coupling between all branches 1-4. We clearly observe that the frequency of the Fano resonance redshifts and the asymmetric profile weakens for large random degree. However, this behavior can essentially be attributed to the weakening of the dark mode alone as already shown in Fig. 5(d). Since the Fano resonance is still guaranteed by the band inversion, the asymmetric Fano shape remains before disappearing, which is an essential difference from trivial Fano resonance [31].

The explanation of these results in terms of symmetryprotected topological states can be inspired by the work in Ref. [35] dealing with acoustic waveguides, while some differences need also to be highlighted. In the latter reference it is shown that the topological modes in one-dimensional multiple scattering systems are symmetry protected, namely, that the topology protects only against disorder that does not change the individual scattering matrix of a pillar for the relevant excitation. This can apply to the disorder along the $x$ axis where each type of mode keeps its symmetry and can be treated independently of each other. On the other hand, the disorder along the $y$ axis partly breaks the symmetry of the modes and produces a coupling between different modes, which means the scattering matrix displays at least two ingoing and outgoing channels. This is a general property of elastic systems, in contrast to acoustic waveguides, owing to the existence of several types of vibrational modes. Therefore, it is likely that the symmetry protection is lost, resulting in the vanishing of the Fano resonance, while the bulk band gap is not yet closed.

\section{SUMMARY}

In summary, we endowed the conventional Fano resonance with topological properties in a pillared phononic beam, the shear motions of which exhibit symmetric and antisymmetric modes. Thanks to a proper design of the structure, double Dirac cones appear in the dispersion of both, which can further be degenerated to form a common topological band gap by band inversion. A high- $Q$ dark edge mode and a low- $Q$ bright edge mode with topological protection are further designed with topological and trivial bulk pillared phononic beams. A topological Fano resonance with extrahigh quality factor is therefore induced from the coupling of the two edge modes. This resonance exhibits strong robustness against random perturbation in the pillars' position. It is found that the robustness of the Fano resonance originates both from band inversion and from symmetry protection of vibrating modes associated to geometry. If both protections are conserved, the asymmetric shape as well as the frequency of the Fano resonance are kept unchanged. On the other hand, if vibration symmetry is broken and only band inversion remains, the asymmetric shape weakens but keeps the shape until disappearing before the closing of the bulk band gap. The frequency of the topological Fano resonance is insensitive to random perturbation along the beam's longitudinal direction whereas a slight frequency shift appears when introducing any transverse random perturbation. The realization of topological high- $Q$ resonance in solids may open a route for many applications in wave functional devices in nano- and microscales such as sensing and information processing [36].

\section{ACKNOWLEDGMENTS}

This work was supported by the National Natural Science Foundation of China (Grant No. 11902223), the Shanghai Pujiang Program (Grant No. 19PJ1410100), the program for professor of special appointment (Eastern Scholar) at Shanghai Institutions of Higher Learning, and a start-up funding from Tongji University. Two of the authors (B.B. and B.D.R.) would like to thank the support and hospitality of Professor Yabin Jin and Tongji University during their visit under the High-Level Foreign Expert Program.
[1] U. Fano, Effects of configuration interaction on intensities and phase shifts, Phys. Rev. 124, 1866 (1961).

[2] B. Luk'yanchuk, N. I. Zheludev, S. A. Maier, N. J. Halas, P. Nordlander, H. Giessen, and C. T. Chong, The Fano resonance in plasmonic nanostructures and metamaterials, Nat. Mater. 9, 707 (2010).

[3] A. E. Miroshnichenko, S. Flach, and Y. S. Kivshar, Fano resonances in nanoscale structures, Rev. Mod. Phys. 82, 2257 (2010).

[4] A. B. Khanikaev, C. Wu, and G. Shvets, Fano-resonant metamaterials and their applications, Nanophotonics 2, 247 (2013).

[5] C. Goffaux, J. Sánchez-Dehesa, A. L. Yeyati, P. Lambin, A. Khelif, J. O. Vasseur, and B. Djafari-Rouhani, Evidence of
Fano-Like Interference Phenomena in Locally Resonant Materials, Phys. Rev. Lett. 88, 225502 (2002).

[6] Y. A. Kosevich, C. Goffaux, and J. Sánchez-Dehesa, Fano-like resonance phenomena by flexural shell modes in sound transmission through two-dimensional periodic arrays of thin-walled hollow cylinders, Phys. Rev. B. 74, 012301 (2006).

[7] K. Lodewijks, J. Ryken, W. Van Roy, G. Borghs, L. Lagae, and P. Van Dorpe, Tuning the Fano resonance between localized and propagating surface plasmon resonances for refractive index sensing applications, Plasmonics 8, 1379 (2013).

[8] Z. L. Sámson, K. F. MacDonald, F. De Angelis, B. Gholipour, K. Knight, C. C. Huang, E. Di Fabrizio, D. W. Hewak, and N. I. Zheludev, Metamaterial electro-optic switch of nanoscale thickness, Appl. Phys. Lett. 96, 143105 (2010). 
[9] M. J. Dicken, K. Aydin, I. M. Pryce, L. A. Sweatlock, E. M. Boyd, S. Walavalkar, J. Ma, and H. A. Atwater, Frequency tunable near-infrared metamaterials based on $\mathrm{VO}_{2}$ phase transition, Opt. Express 17, 18330 (2009).

[10] J. Chen, Z. Li, J. Li, and Q. Gong, Compact and high-resolution plasmonic wavelength demultiplexers based on Fano interference, Opt. Express 19, 9976 (2011).

[11] Z. Chen, R. Hu, L. Cui, L. Yu, L. Wang, and J. Xiao, Plasmonic wavelength demultiplexers based on tunable Fano resonance in coupled-resonator systems, Opt. Commun. 320, 6 (2014).

[12] S.-L. Chua, Y. Chong, A. D. Stone, M. Soljačić, and J. BravoAbad, Low-threshold lasing action in photonic crystal slabs enabled by Fano resonances, Opt. Express 19, 1539 (2011).

[13] N. Papasimakis and N. I. Zheludev, Metamaterial-induced transparency: Sharp Fano resonances and slow light, Opt. Photon. News 20, 22 (2009).

[14] M. Z. Hasan and C. L. Kane, Colloquium: Topological insulators, Rev. Mod. Phys. 82, 3045 (2010).

[15] M. C. Rechtsman, J. M. Zeuner, Y. Plotnik, Y. Lumer, D. Podolsky, F. Dreisow, S. Nolte, M. Segev, and A. Szameit, Photonic Floquet topological insulators, Nature 496, 196 (2013).

[16] A. B. Khanikaev, S. H. Mousavi, W.-K. Tse, M. Kargarian, A. H. MacDonald, and G. Shvets, Photonic topological insulators, Nat. Mater. 12, 233 (2013).

[17] Z. Yang, F. Gao, X. Shi, X. Lin, Z. Gao, Y. Chong, and B. Zhang, Topological Acoustics, Phys. Rev. Lett. 114, 114301 (2015).

[18] X. Zhang, M. Xiao, Y. Cheng, M.-H. Lu, and J. Christensen, Topological sound, Commun. Phys. 1, 97 (2018).

[19] R. Fleury, A. B. Khanikaev, and A. Alu, Floquet topological insulators for sound, Nat. Commun. 7, 11744 (2016).

[20] J. Lu, C. Qiu, L. Ye, X. Fan, M. Ke, F. Zhang, and Z. Liu, Observation of topological valley transport of sound in sonic crystals, Nat. Phys. 13, 369 (2017).

[21] Y.-G. Peng, C.-Z. Qin, D.-G. Zhao, Y.-X. Shen, X.-Y. Xu, M. Bao, H. Jia, and X.-F. Zhu, Experimental demonstration of anomalous Floquet topological insulator for sound, Nat. Commun. 7, 13368 (2016).

[22] A. Souslov, B. C. Van Zuiden, D. Bartolo, and V. Vitelli, Topological sound in active-liquid metamaterials, Nat. Phys. 13, 1091 (2017).
[23] G. Ma, M. Xiao, and C. Chan, Topological phases in acoustic and mechanical systems, Nat. Rev. Phys. 1, 281 (2019).

[24] S. H. Mousavi, A. B. Khanikaev, and Z. Wang, Topologically protected elastic waves in phononic metamaterials, Nat. Commun. 6, 8682 (2015).

[25] Y. Ding, Y. Peng, Y. Zhu, X. Fan, J. Yang, B. Liang, X. Zhu, X. Wan, and J. Cheng, Experimental Demonstration of Acoustic Chern Insulators, Phys. Rev. Lett. 122, 014302 (2019).

[26] H. Chen, L. Yao, H. Nassar, and G. Huang, Mechanical Quantum Hall Effect in Time-Modulated Elastic Materials, Phys. Rev. Appl. 11, 044029 (2019).

[27] M. Miniaci, R. Pal, B. Morvan, and M. Ruzzene, Experimental Observation of Topologically Protected Helical Edge Modes in Patterned Elastic Plates, Phys. Rev. X 8, 031074 (2018).

[28] Y. Jin, D. Torrent, and B. Djafari-Rouhani, Robustness of conventional and topologically protected edge states in phononic crystal plates, Phys. Rev. B 98, 054307 (2018).

[29] W. Wang, B. Bonello, B. Djafari-Rouhani, and Y. Pennec, Topological valley, pseudospin, and pseudospin-valley protected edge states in symmetric pillared phononic crystals, Phys. Rev. B 100, 140101 (2019).

[30] M. Miniaci, R. K. Pal, R. Manna, and M. Ruzzene, Valley-based splitting of topologically protected helical waves in elastic plates, Phys. Rev. B 100, 024304 (2019).

[31] F. Zangeneh-Nejad and R. Fleury, Topological Fano Resonances, Phys. Rev. Lett. 122, 014301 (2019).

[32] Y. Pennec, Y. Jin, and B. Djafari-Rouhani, Phononic and photonic crystals for sensing applications, Adv. Appl. Mech. 52, 105 (2019).

[33] R. Chaunsali, C.-W. Chen, and J. Yang, Subwavelength and directional control of flexural waves in zone-folding induced topological plates, Phys. Rev. B 97, 054307 (2018).

[34] Y. Chen, X. Liu, and G. Hu, Topological phase transition in mechanical honeycomb lattice, J. Mech. Phys. Solids 122, 54 (2019).

[35] F. Zangeneh-Nejad and R. Fleury, Topological analog signal processing, Nat. Commun. 10, 2058 (2019).

[36] J. Jin, X. Yin, L. Ni, M. Soljačić, B. Zhen, and C. Peng, Topologically enabled ultrahigh-Q guided resonances robust to out-of-plane scattering, Nature 574, 501 (2019). 